\title{
Evaluation of aortic stenosis using cardiovascular magnetic resonance: a systematic review \& meta-analysis
}

\author{
Kei Woldendorp ${ }^{1,2,3,4}$ (D), Paul G. Bannon ${ }^{1,2,3}$ and Stuart M. Grieve ${ }^{1,2,5^{*}}$
}

\begin{abstract}
Background: As the average age of patients with severe aortic stenosis (AS) who receive procedural intervention continue to age, the need for non-invasive modalities that provide accurate diagnosis and operative planning is increasingly important. Advances in cardiovascular magnetic resonance (CMR) over the past two decades mean it is able to provide haemodynamic data at the aortic valve, along with high fidelity anatomical imaging.

Methods: Electronic databases were searched for studies comparing CMR to transthoracic echocardiography (TTE) and transoesophageal echocardiography (TEE) in the diagnosis of AS. Studies were included only if direct comparison was made on matched patients, and if diagnosis was primarily through measurement of aortic valve area (AVA).

Results: Twenty-three relevant, prospective articles were included in the meta-analysis, totalling 1040 individual patients. There was no significant difference in AVA measured as by CMR compared to TEE. CMR measurements of AVA size were larger compared to TTE by an average of $10.7 \%$ (absolute difference: $+0.14 \mathrm{~cm}^{2}, 95 \% \mathrm{Cl} 0.07-0.21, p<0.001$ ). Reliability was high for both inter- and intra-observer measurements $\left(0.03 \mathrm{~cm}^{2}+/-0.04\right.$ and $0.02 \mathrm{~cm}^{2}+/-0.01$, respectively).

Conclusions: Our analysis demonstrates the equivalence of AVA measurements using CMR compared to those obtained using TEE. CMR demonstrated a small but significantly larger AVA than TTE. However, this can be attributed to known errors in derivation of left ventricular outflow tract size as measured by TTE. By offering additional anatomical assessment, CMR is warranted as a primary tool in the assessment and workup of patients with severe AS who are candidates for surgical or transcatheter intervention.
\end{abstract}

Keywords: Cardiovascular magnetic resonance, CMR, Aortic valve, Aortic stenosis, Aortic regurgitation, Valve dysfunction

\section{Introduction}

Cardiovascular magnetic resonance imaging (CMR) has, since its introduction in the 1980 s, evolved to become a viable non-invasive alternative to echocardiography for a

\footnotetext{
* Correspondence: stuart.grieve@sydney.edu.au

${ }^{1}$ Sydney Translational Imaging Laboratory, Imaging and Phenotyping Laboratory, Charles Perkins Centre, Faculty of Medicine and Health, The University of Sydney, Camperdown, NSW 2006, Australia

${ }^{2}$ Sydney Medical School, The University of Sydney, Camperdown, NSW 2050, Australia

Full list of author information is available at the end of the article
}

wide variety of cardiac pathology. Aortic stenosis (AS) is a common disease with a devastating clinical impact; without intervention AS progresses inexorably and once symptoms develop the life-expectancy is reduced to an average of 3 years unless the mechanical obstruction is not relieved [1]. Transthoracic echocardiography (TTE) is the clinical reference standard - it is rapid, safe, well tolerated by patients and is by far the most common exam used for evaluation of aortic valvular disease. The considerable technical advances in CMR have improved

(c) The Author(s). 2020 Open Access This article is licensed under a Creative Commons Attribution 4.0 International License, which permits use, sharing, adaptation, distribution and reproduction in any medium or format, as long as you give appropriate credit to the original author(s) and the source, provide a link to the Creative Commons licence, and indicate if changes were made. The images or other third party material in this article are included in the article's Creative Commons licence, unless indicated otherwise in a credit line to the material. If material is not included in the article's Creative Commons licence and your intended use is not permitted by statutory regulation or exceeds the permitted use, you will need to obtain permission directly from the copyright holder. To view a copy of this licence, visit http://creativecommons.org/licenses/by/4.0/ The Creative Commons Public Domain Dedication waiver (http://creativecommons.org/publicdomain/zero/1.0/) applies to the data made available in this article, unless otherwise stated in a credit line to the data. 
the quality of the anatomical and functional information available from CMR. Here, we systematically the current available evidence regarding the use of CMR compared to echocardiography to evaluate the clinical status and practicality of this technique [2].

Diagnosis of AS, and particularly severe AS is determined by a combination of mean and peak pressure gradients across the valve as well as the effective valve orifice, or aortic valve area (AVA) [2]. Based on these criteria severe AS is specified by an AVA of $<1 \mathrm{~cm}^{2}$ determined by the maximum opening of the aortic valve during systole [3]. Several methods have been designed to calculate this, including the Gorlin formula [4] for use with invasive cardiac catheterisation (now seldom used), the continuity equation [5] used with TTE and determined on a series of measurements including the left ventricular outflow tract (LVOT), and planimetry [6] and computed tomography (CT) that determine AVA by direct measurement of the valve orifice.

CMR has emerged as an alternate, non-invasive method, and offers prognostic and planning potential for procedural intervention in the form of transcatheter aortic valve implantation (TAVI) or surgical aortic valve replacement (SAVR). Additionally, CMR provides high fidelity anatomical imaging, thus avoiding the need for additional imaging tools such as CT in the workup for valve intervention. This may be particularly beneficial in patients with impaired renal function where high iodinated contrast loads required for CT imaging are contraindicated [7]. Contrast enhanced CMR can provide valuable information on anatomy and myocardial scar, however gadolinium contrast is not required for basic functional assessment or for valve flow quantification [8].

The rapid recent growth in volume of aortic interventional procedures is increasing demand for accurate assessment of aortic valve anatomy and function. CMR represents an attractive non-invasive method in view of the lack of radiation exposure, the relative safety of nonionic gadolinium agents relative to iodine contrast, and the ability of CMR to provide a mix of cross-sectional anatomical, flow and ventricular functional information. This paper aims to review the current literature on use of CMR for aortic valve assessment in comparison to both TTE and transesophageal echocardiography (TEE).

\section{Methods}

Following the PRISMA (Preferred Reporting Items for Systematic Reviews and Meta-Analysis) guidelines, electronic searches were conducted by two authors (KW + SG). Electronic searches were performed using Web of Knowledge, Embase, and PubMed. Keywords included in the search were "CMR", "cardiac magnetic resonance", "aorta", "aortic valve" "aortic root" and "aortic pathology". Eligible studies were prospective studies published in English that compared CMR with either TTE or TEE for AVA evaluation. The reference lists of all retrieved articles were reviewed for further identification of potentially relevant studies (Fig. 1). All data were extracted from article texts, tables, figures, and appendices. Articles included were assessed for quality using the Newcastle-Ottawa Quality Assessment Scale (NOS) (Supplementary).

\section{Inclusion criteria}

Only studies that specified either measured or derived AVA (as opposed to correlations between imaging methods) were included in the meta-analysis portion of the review.

\section{Analysis}

Baseline characteristics and intervention details were presented as raw values (\%) or mean \pm standard deviation unless otherwise indicated. Pooled values for clinical outcomes were reported as mean \pm standard deviation or as otherwise specified. Data were summarized as standard mean difference, with overall weighted mean presented where appropriate. $\mathrm{I}^{2}$ statistic was used to estimate the percentage of total variation across studies, due to heterogeneity rather than chance. An $\mathrm{I}^{2}$ value of greater than $50 \%$ was considered substantial heterogeneity. If there was substantial heterogeneity, the possible clinical and methodological reasons for this were explored qualitatively. All $P$-values were 2 -sided. A significant difference was defined as $P<0.05$ (without correction for multiplicity). Statistical analysis was conducted with Review Manager Version 5.3 (Cochrane Collaboration, Software Update, Oxford, UK).

\section{Results}

A total of 2623 publications were identified through two online database searches and from collated reference lists (Fig. 1). After exclusion of duplicated or irrelevant publications, a total of 23 relevant, prospective articles were included in the meta-analysis, totalling 1040 individual patients [9-31]. One study which investigated the use of CMR in subsets of low flow/low gradient AS (59 patients of 128 total) was separated into four discrete datasets for the meta-analysis [9] (Table 1). AS was identified in 659 (63.4\%) patients, aortic regurgitation (AR) in $26(2.2 \%)$, mixed pathology in $74(6.3 \%)$, and controlled subjects numbered 294 (28.3\%).

The vast majority of measurements were done using a 1.5 T CMR (1030 subjects, 99.1\%) patients, a $3 \mathrm{~T}$ CMR was used in only $10(0.9 \%)$ patients. CMR protocols used included balanced steady-state free procession (bSSFP) in 967 (93.0\%) patients and gradient echo (GRE) in 73 (7.0\%) patients. Where multiple protocols were used, the one closest to the study result was used in the meta- 


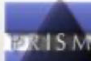

PRISMA 2009 Flow Diagram
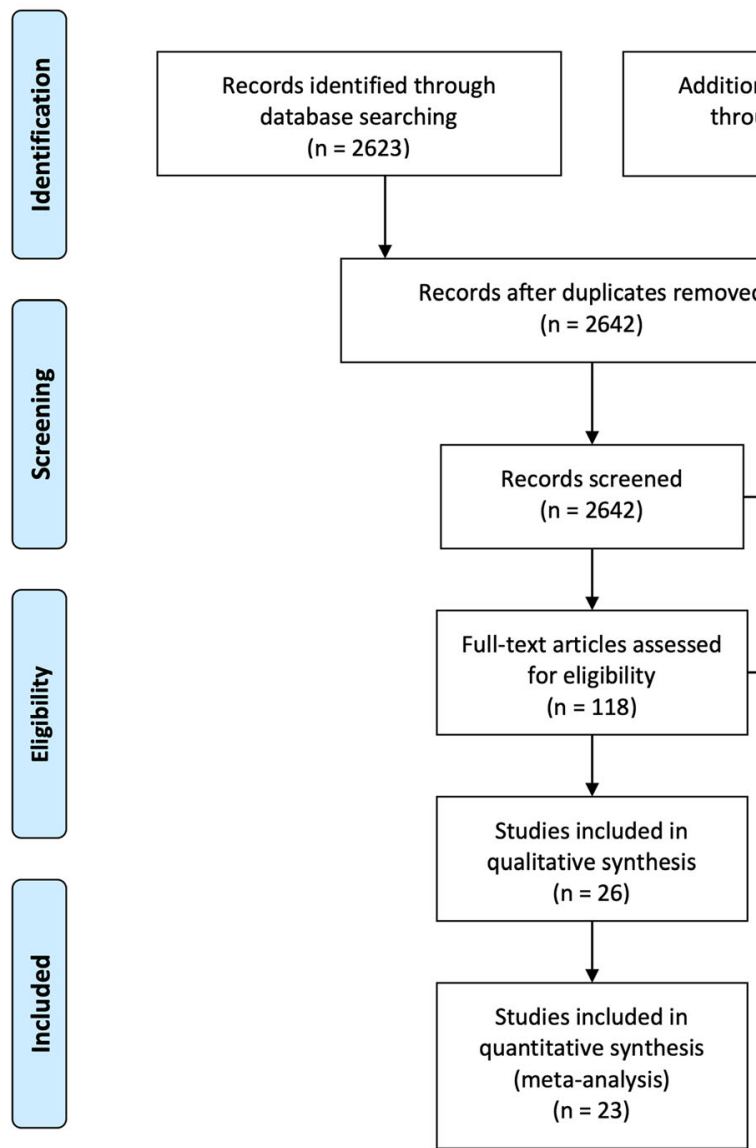

al records identifie through other sources $(n=19)$
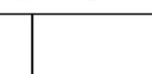
Table 1 Summary of papers included in the meta-analysis

\begin{tabular}{|c|c|c|c|c|c|c|c|c|c|c|c|c|}
\hline \multirow[b]{2}{*}{ Author } & \multirow[b]{2}{*}{ Year } & \multirow[b]{2}{*}{$n$} & \multirow[b]{2}{*}{ Males } & \multirow[b]{2}{*}{ Age (yrs) } & \multicolumn{3}{|c|}{ Pathology } & \multicolumn{3}{|l|}{ AVA $\left(\mathrm{cm}^{2}\right)$} & \multicolumn{2}{|l|}{ Reliability (CMR) } \\
\hline & & & & & AS & $A R$ & Mixed & CMR & TTE & TEE & Inter & Intra \\
\hline $\begin{array}{l}\text { Barone-Rochette } \\
\mathrm{HG} / \mathrm{NF}\end{array}$ & 2013 & 69 & 43 & $75 \pm 10$ & 69 & & & $1.0 \pm 0.02$ & $0.71 \pm 0.13$ & & & \\
\hline $\begin{array}{l}\text { Barone-Rochette } \\
\text { HG/LF }\end{array}$ & 2013 & 28 & 16 & $72 \pm 14$ & 28 & & & $0.9 \pm 0.1$ & $0.54 \pm 0.12$ & & & \\
\hline $\begin{array}{l}\text { Barone-Rochette LG/ } \\
\text { NF }\end{array}$ & 2013 & 17 & 8 & $72 \pm 9$ & 17 & & & $1.2 \pm 0.2$ & $0.84 \pm 0.09$ & & & \\
\hline $\begin{array}{l}\text { Barone-Rochette LG/ } \\
\text { LF }\end{array}$ & 2013 & 14 & 8 & $72 \pm 6$ & 14 & & & $1.0 \pm 0.2$ & $0.81 \pm 0.15$ & & & \\
\hline Buchner & 2015 & 8 & 1 & & 8 & & & $0.75 \pm 0.09$ & $0.69 \pm 0.07$ & $\begin{array}{l}0.79 \pm \\
0.15\end{array}$ & $6 \%$ & $3 \%$ \\
\hline Debl & 2005 & 33 & & & & & & $0.94 \pm 0.29$ & $0.85 \pm 0.31$ & & & \\
\hline Defrance & 2012 & 74 & 33 & $75 \pm 14$ & 43 & & & $\begin{array}{l}1.00(0.80- \\
2.30)\end{array}$ & $\begin{array}{l}0.93(0.68- \\
2.42)\end{array}$ & & $1.80 \pm 2.27 \%$ & \\
\hline Dimitriou & 2012 & 14 & & & 14 & & & $1.0 \pm 0.4$ & $1.0 \pm 0.2$ & & & \\
\hline Friedrich & 2001 & 25 & & $64 \pm 8$ & 15 & & 10 & $0.86 \pm 0.25$ & $0.79 \pm 0.2$ & & $15.20 \%$ & $17.80 \%$ \\
\hline Garcia & 2013 & 68 & 39 & $64 \pm 15$ & 60 & & & $1.4 \pm 0.41$ & $1.19 \pm 0.28$ & & & \\
\hline John & 2003 & 40 & 25 & $70 \pm 8.8$ & 40 & & & $0.91 \pm 0.25$ & & $\begin{array}{l}0.89 \pm \\
0.28\end{array}$ & $0.07 \pm 0.06$ & $0.05 \pm 0.04$ \\
\hline $\begin{array}{l}\text { Knobelsdorff- } \\
\text { Brenkenhoff }\end{array}$ & 2009 & 65 & & & 2 & 1 & & $1.71 \pm 0.46$ & $1.7 \pm 0.4$ & $\begin{array}{l}1.82 \pm \\
0.53\end{array}$ & $11.5 \pm 7.8 \%$ & $6.7 \pm 5.4 \%$ \\
\hline Kupfahl & 2003 & 44 & 27 & & 44 & & & $0.8 \pm 0.25$ & $0.7 \pm 0.3$ & $\begin{array}{l}0.8 \pm \\
0.28\end{array}$ & $0.03 \pm 0.05$ & $-0.02 \pm 0.06$ \\
\hline Levy & 2016 & 91 & 60 & $74 \pm 10$ & 91 & & & $0.9 \pm 0.22$ & $0.81 \pm 0.18$ & & $0.83[0.42-0.95]$ & 0.82 [0.39-0.95] \\
\hline Malyar & 2008 & 42 & 17 & $71 \pm 8$ & 20 & & 22 & $0.97 \pm 0.3^{a}$ & $0.75 \pm 0.28^{b}$ & $\begin{array}{l}0.87 \pm \\
0.25^{c}\end{array}$ & & \\
\hline Mutnuru & 2016 & 50 & & & 10 & 9 & 8 & $1.12 \pm 0.25$ & $1.10 \pm 0.21$ & & & \\
\hline O'Brien & 2009 & 15 & & & 15 & & & $0.85 \pm 0.3$ & & $\begin{array}{l}0.85 \pm \\
0.24\end{array}$ & & \\
\hline Paelinck & 2011 & 24 & 8 & $\begin{array}{l}83.5(67- \\
88)\end{array}$ & 24 & & & $\begin{array}{l}0.60(0.3- \\
0.8)\end{array}$ & $\begin{array}{l}0.54(0.32- \\
0.83)\end{array}$ & $\begin{array}{l}0.6 \\
(0.37- \\
0.8)\end{array}$ & & \\
\hline Pontone & 2013 & 50 & 27 & $79.6 \pm 7.5$ & 50 & & & $0.4 \pm 0.1$ & $0.4 \pm 0.1$ & & & \\
\hline Pouleur & 2007 & 48 & 33 & $62 \pm 13$ & 27 & & & $2.4 \pm 1.8$ & $2.0 \pm 1.5$ & $\begin{array}{l}2.5 \pm \\
1.7\end{array}$ & $0.1 \pm 0.3$ & $0.0 \pm 0.3$ \\
\hline Reant & 2006 & 39 & 25 & $71.1 \pm 7.6$ & 13 & & 26 & $0.92 \pm 0.29$ & $0.75 \pm 0.28$ & $\begin{array}{l}0.93 \pm \\
0.31\end{array}$ & $0.03 \pm 0.14 \mathrm{~cm} 2$ & $0.02 \pm 0.07 \mathrm{~cm} 2$ \\
\hline Speiser & 2014 & 48 & 30 & $64 \pm 18$ & 23 & & & $1.9 \pm 1.1$ & $1.7 \pm 0.8$ & & $\begin{array}{l}0.027 \pm 0.13 \mathrm{~cm} 2 \\
(0.53 \%)\end{array}$ & $\begin{array}{l}0.027 \pm 0.06 \mathrm{~cm} 2 \\
(0.69 \%)\end{array}$ \\
\hline Weininger & 2011 & 22 & 13 & & 22 & & & $0.65 \pm 0.34$ & & $\begin{array}{l}0.78 \pm \\
0.15\end{array}$ & $0.01 \pm 0.03$ & $0.01 \pm 0.02$ \\
\hline Westermann & 2011 & 27 & 16 & $61.8 \pm 8.3$ & 18 & 1 & & $1.04 \pm 0.39^{d}$ & $0.88 \pm 0.22$ & & $4.3 \pm 2.6 \%$ & $2.9 \pm 1.0 \%$ \\
\hline
\end{tabular}

$A R$ Aortic regurgitation, AS Aortic stenosis, CMR Cardiovascular magnetic resonance, HG/NF High-gradient/normal-flow, HG/LF High-gradient/low-flow, LG/NF Lowgradient/normal-flow, LG/LF Low-gradient/low-flow, TEE Transesophageal echocardiography, TTE Transthoracic echocardiography

anly completed in 26 patients

bonly completed in 41 patients

conly completed in 38 patients

donly for patients with AS 


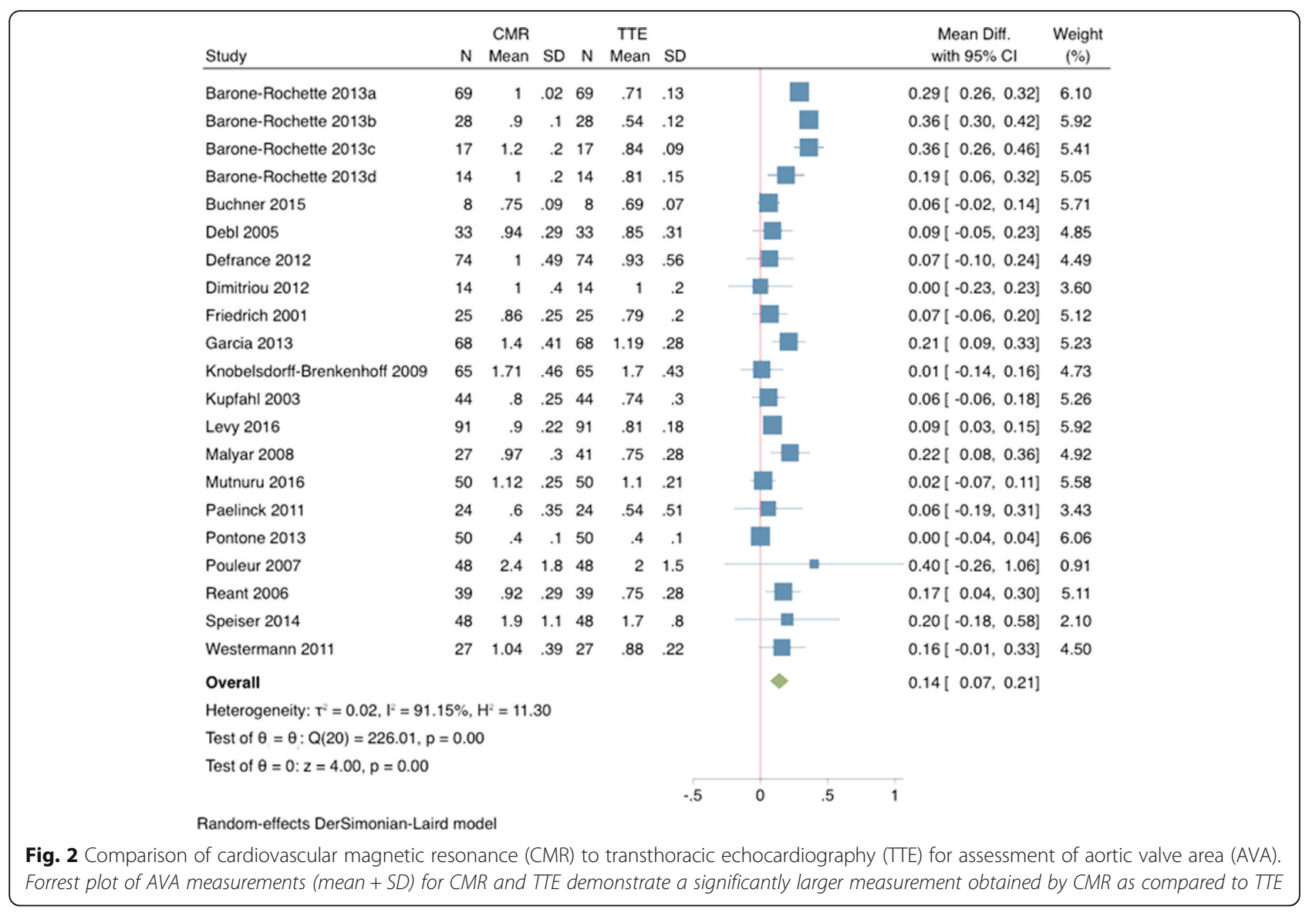

from CMR overestimate those from TTE by an average of $0.17 \mathrm{~cm}^{2}$.

A standard for measurement of AS is cardiac catheterisation, but due to its invasive nature and risk of potential stroke is rarely performed in favour of less invasive and safer procedures. TTE is the clinical reference standard, however previous studies have shown that CMR performs as well as, or better than, TTE or TEE compared to direct catheterisation in the assessment of aortic stenosis [20, 28].

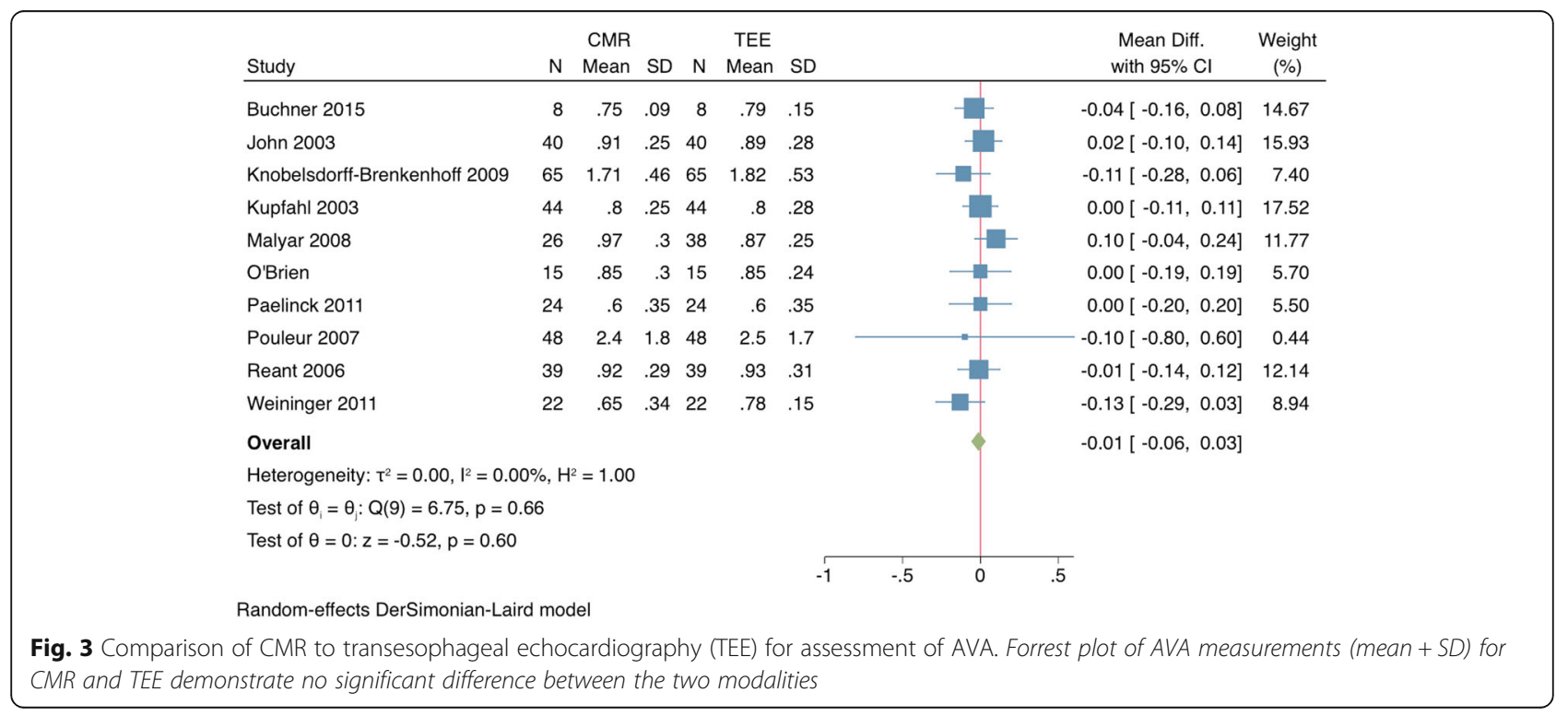


Table 2 Comparison of haemodynamic measurements between CMR and TTE

\begin{tabular}{|c|c|c|c|c|c|}
\hline Author & Year & $\mathrm{n}$ & AS & Method & Conclusion \\
\hline Defrance & 2012 & 74 & 53 & $\begin{array}{l}\text { Novel semi-automated process as de- } \\
\text { scribed by Bollache, et al. } 2010\end{array}$ & $\begin{array}{l}\text { Good agreement with TTE for PV and MG }(r=0.92 \text { with mean bias }-0.29 \pm \\
0.62 \mathrm{~m} / \mathrm{s} \text { and } r=0.86 \text { with mean bias }-12 \pm 15 \mathrm{mmHg} \text {, respectively })\end{array}$ \\
\hline Caruthers & 2002 & 24 & 24 & $\begin{array}{l}\text { Simpsons rule for VTI and modified } \\
\text { Bernoulli equation for gradients }\end{array}$ & Good agreement with TTE for PG and MG ( $r=0.83$ and $r=0.87$, respectively) \\
\hline Levy & 2016 & 91 & 91 & Not described & Good agreement with TTE for PV ( $r=0.73$ with mean bias $-0.35 \pm 0.40 \mathrm{~m} / \mathrm{s})$ \\
\hline Garcia & 2013 & 68 & 60 & Simplified Bernoulli equation & Good agreement with TTE for MG ( $r=0.7$ with mean bias $-2.8 \mathrm{mmHg})$ \\
\hline Eichenberger & 1993 & 19 & 19 & Simplified Bernoulli equation & Good agreement with TTE for MG $(r=0.96)$ \\
\hline Sirin & 2014 & $19^{\mathrm{a}}$ & 19 & Simplified Bernoulli equation & Good agreement with TTE for MG $(r=0.84$ with mean bias $-12.4 \mathrm{mmHg})$ \\
\hline
\end{tabular}

${ }^{\text {a Paediatric patients }}$

PV Peak velocity, MG Mean gradient, PV Peak gradient, VTI Velocity time interval

The AVA measurement from TTE assessment is derived using the continuity equation and is based on the ratio between Doppler stroke volume and post-aortic valve flow. Calculation of Doppler stroke volume relies on an accurate estimation of the LVOT, a value that is then squared in the continuity equation formula. In TTE this is done at one measurement, in the parasternal long-axis view, on the assumption that the LVOT is circular. However experience from TAVI valve sizing highlights that the LVOT is frequently elliptical and thus measurements from TTE may underestimate or underestimate the true LVOT size, and subsequently also underestimate AVA $[32,33]$ or overestimated AVA. This was confirmed in a paper by Chin et al., which demonstrated that TTE underestimated AVA by $0.23 \mathrm{~cm}^{2}$ compared with CMR [34]. Similar discrepancies in calculated AVA have been noted in other studies comparing CT to TTE and TEE to TTE for AVA measurements $[35,36]$. The discrepancies noted have significant implication on AS grading and provide an explanation for the so-named "paradoxical low-flow, low-gradient severe AS" [34]. Furthermore, use of the continuity equation creates further confoundment in the form of pressure recovery estimation errors of AS severity. In a large cohort study of over 1000 patients it was noted that with specific pressure recovery adjustment almost $50 \%$ of severe AS patients were reclassified as non-severe [37]. The ability of CMR to assess complete cardiac function, including blood velocity in multiple sites simultaneously, makes correction of pressure recovery discrepancies far easier and less prone to user error [38, 39].

For patients with low gradient severe aortic stenosis. There was again good correlation between TTE and CMR values for measured LVOT and AVA. CMR calculated AVA slightly larger in patients with low gradient severe aortic stenosis compared to high gradient severe AS, however as mentioned above this is likely due to estimation errors derived from TTE [9]. This highlights a major advantage of CMR over echocardiography for anatomical based diagnosis of patients with low flow low gradient severe aortic stenosis.
Patients with severe AS who are TAVI candidates need accurate anatomical assessments of their LVOT, aortic annulus size, coronary anatomy, and ascending aorta. Studies have demonstrated that CMR produces values in keeping with those seen in CT and TEE for morphological assessments $[26,40,41]$. CT is able to provide precise evaluation of vascular and annular calcification, as well as routine vessel size measurements - facilitating interventional planning. CMR could potentially be used similarly but this is not currently widely practiced. However CMR is the gold standard for functional evaluation of the left ventricle and, unlike CT, can identify the pathological effects of left ventricular remodelling, particularly subendocardial fibrosis, which have been implicated in prognosis post AVR or TAVI [30, 42].

CMR allows for more accurate assessment of valve pathology because it can render images in any plane, however this comes at the price of some loss finer detail due to thicker imaging slices in lower powered machines [43]. Nevertheless, studies have demonstrated higher sensitivity and specificity of CMR to identify correct valvular pathology when compared with traditional methods and confirmed pathologically [11, 44, 45]. Because of the ability of CMR to measure time-resolved, cross-sectional flow (as opposed to inferred flow rates derived in Doppler echocardiography) it may also provide benefit in patients with complex aortic morphology not amenable to echocardiographic assessment [43].

CMR also avoids many of the pitfalls of other imaging techniques including: unnecessary sedation/anaesthesia for TEE and contrast exposure of CT. Gadolinium contrast is not necessary for evaluation of function using cine CMR sequences or for quantification of flow using phase contrast techniques. Avoiding the contrast loading of CT is often cited as another potential benefit of CMR. Currently guidelines still advocate caution and most suggest an estimated glomerular filtration rate $(\mathrm{eGFR})<30$ $\mathrm{ml} / \mathrm{min} / 1.73 \mathrm{~m}^{2}$ be a cut-off for patients who are at increased risk of contrast induced nephropathy [7]. Considering that most patients with severe AS are elderly, and eGFR has a natural decline with age, this presents 
significant implications to anatomical screening in prospective patients with iodine contrast reliant methods.

CMR has the potential for accurate haemodynamic measurements of the cardiovascular system [8, 46-48]. However, this is not standard practice and significant heterogeneity exists in the algorithms used to calculate transvalvular aortic gradients. The prevailing evidence in the literature suggests that current clinical CMR protocols give reasonably good correlation to TTE haemodynamic measurements but in general will underestimate these to a variable degree $[13,17,21,49,50]$. The presence of turbulent flow present challenges for both echocardiography and CMR measurements and accounts for some differences between [17]. Turbulent flow results in signal loss during CMR due to intravoxel dephasing, which may lead to inaccurate measurements of effective orifice area boundaries [51]. Peak gradient estimation in echocardiography is directly affected by turbulent flow since the Bernouli equation can underestimate the true pressure difference by not accounting for flow turbulence, and overestimate the total pressure loss by neglecting the effect of pressure recovery in the post-stenotic region $[17,52]$. CMR evaluation of flow at extremely high flow is known to underestimate velocity measurements, secondary to intravoxel dephasing errors [25], although improvements in CMR hardware have improved performance through reductions in echo time (TE) and secondary eddy currents [53]. Advanced methods such as adaptive valve plane phase-contrast CMR and 4D-flow CMR are now clinically feasible, but have not yet translated to the clinical setting, and will need clinicals familiar to their protocols to become used in general clinical applications.

\section{Conclusion}

Our analysis demonstrates the equivalence of valve area measurements in AS using CMR compared to those obtained using TEE. A small but significance difference was noted between CMR and TTE, where higher values found using CMR. Prior data shows that TTE underestimates AVA calculated from LVOT area compared to TEE, attributed to incomplete visualisation of the nonuniform anatomy of the aortic valve. Evidence relating to aortic velocity and pressure gradient measurements are highly heterogeneous, reflecting the complex and evolving nature of CMR flow technology. Given the equivalence of CMR AVA to the clinical reference standard, the additional benefits of CMR may increase the value proposition of this technique for pre-operative workup. Although TTE is rapid, safe, and well tolerated by patients, the ability of CMR to accurately image the remainder of the cardiovascular system, warrant its inclusion in the broader assessment and surgical workup of patients with AS.

\section{Abbreviations}

AR: Aortic regurgitation; AS: Aortic stenosis; AVA: Aortic valve area; bSSFP: Balanced steady state free precession; CMR: Cardiovascular magnetic resonance; CT: Computed tomography; eGFR: Estimated glomerular filtration rate; LVOT: Left ventricular outflow tract; MG: Mean gradient; NOS: Newcastle-Ottawa Quality Assessment scale; PG: Peak gradient; PRISMA: Preferred Reporting Items for Systematic Reviewrs and MetaAnalysis; PV: Peak velocity; SAVR: Surgical aortic valve replacement; TAVI: Transcatheter aortic valve implantation; TE: Echo time; TEE: Transesophageal echocardiography; TTE: Transthoracic echocardiography

\section{Acknowledgements}

N/a

\section{Authors' contributions}

KW did the literature review, extracted data, analysed data, wrote and prepared the manuscript and all tables and figures. PB and SG were both responsible as research supervisors as well as providing significant input to the design and edits of the manuscript drafts. The author(s) read and approved the final manuscript.

\section{Funding}

Not applicable.

\section{Availability of data and materials}

Not applicable.

Ethics approval and consent to participate

Not applicable.

\section{Consent for publication}

Not applicable.

\section{Competing interests}

Not applicable.

\section{Author details}

${ }^{1}$ Sydney Translational Imaging Laboratory, Imaging and Phenotyping Laboratory, Charles Perkins Centre, Faculty of Medicine and Health, The University of Sydney, Camperdown, NSW 2006, Australia. ${ }^{2}$ Sydney Medical School, The University of Sydney, Camperdown, NSW 2050, Australia. ${ }^{3}$ Baird Institute of Applied Heart \& Lung Surgical Research, Newtown, NSW 2042, Australia. ${ }^{4}$ Department of Cardiothoracic Surgery, Royal Prince Alfred Hospital, Camperdown, NSW 2006, Australia. ${ }^{5}$ Department of Radiology, Royal Prince Alfred Hospital, Camperdown, NSW 2006, Australia.

Received: 24 June 2019 Accepted: 8 May 2020

Published online: 15 June 2020

\section{References}

1. Carabello BA. Introduction to aortic stenosis. Circ Res. 2013;113(2):179-85 American Heart Association, Inc.

2. Nishimura RA, Otto CM, Bonow RO, Carabello BA, Erwin JP, Guyton RA, et al. AHA/ACC guideline for the management of patients with valvular heart disease: a report of the American College of Cardiology/American Heart Association task force on practice guidelines. J Thoracic Cardiovasc Surg. 2014;148:e1-e132 Elsevier.

3. Sommer G, Bremerich J, Lund G. Magnetic resonance imaging in valvular heart disease: clinical application and current role for patient management. J Magn Reson Imaging. 2012;35(6):1241-52.

4. GORLIN R, GORLIN SG. Hydraulic formula for calculation of the area of the stenotic mitral valve, other cardiac valves, and central circulatory shunts. I American Heart Journal. 1951;41(1):1-29.

5. Otto CM, Pearlman AS, Comess KA, Reamer RP, Janko CL, Huntsman LL. Determination of the stenotic aortic valve area in adults using Doppler echocardiography. JAC. 1986;7(3):509-17.

6. Hoffmann R, Flachskampf FA, Hanrath P. Planimetry of orifice area in aortic stenosis using multiplane transesophageal echocardiography. JAC. 1993; 22(2):529-34. 
7. ACR Manual on Contrast Media. 10 ed. Virginia, USA: ACR Committee on Drugs and Contrast Media; 2017 Jun pp. 1-129.

8. Lawley CM, Broadhouse KM, Callaghan FM, Winlaw DS, Figtree GA, Grieve SM. 4D flow magnetic resonance imaging: role in pediatric congenital heart disease. Asian Cardiovasc Thorac Ann. 2017;26(1):28-37 SAGE PublicationsSage UK: London, England.

9. Barone-Rochette G, Pierard S, Seldrum S, de Meester de Ravenstein C, Melchior J, Maes F, et al. Aortic valve area, stroke volume, left ventricular hypertrophy, remodeling, and fibrosis in aortic stenosis assessed by Cardiac magnetic resonance imaging: comparison between high and low gradient and Normal and low flow aortic stenosis. Circ Cardiovasc Imaging. 2013;6(6): 1009-17.

10. Bruder O, Jochims M, Hunold P, Jensen C, Forsting M, Sabin GV, et al. Comparison of aortic valve area measured by magnetic resonance imaging and dual-source computed tomography. Acta Radiol. 2009; 50(6):645-51.

11. Buchner S, Debl K, Schmid F-X, Luchner A, Djavidani B. Cardiovascular magnetic resonance assessment of the aortic valve stenosis: an in vivo and ex vivo study. BMC Med Imaging. 2015;22:1-8.

12. Debl K, Djavidani B, Seitz J, Nitz W, Schmid FX, Muders F, et al. Planimetry of aortic valve area in aortic stenosis by magnetic resonance imaging. Investig Radiol. 2005;40(10):361-636.

13. Defrance C, Bollache E, Kachenoura N, Perdrix L, Hrynchyshyn N, Bruguiere $E$, et al. Evaluation of aortic valve stenosis using cardiovascular magnetic resonance: comparison of an original Semiautomated analysis of phaseContrast cardiovascular magnetic resonance with Doppler echocardiography. Circ Cardiovasc Imaging. 2012;5(5):604-12.

14. Dimitriou P, Kahari A, Emilsson K, Thunberg P. Cardiovascular magnetic resonance imaging and transthoracic echocardiography in the assessment of stenotic aortic valve area: a comparative study. Acta Radiol. 2012;53(9): 995-1003.

15. Eichenberger AC, Jenni R, Von Schulthess GK. Aortic Valve Pressure Gradients in Patients with Aortic Valve Stenosis. Am J Radiol. 1993;160:971-7.

16. Friedrich MG, Schulz-Menger J, Poetsch T, Pilz B, Uhlich F, Dietz R. Quantification of valvular aortic stenosis by magnetic resonance imaging. Am Heart J. 2002;144(2):329-34.

17. Garcia J, Capoulade R, Le Ven F, Gaillard E, Kadem L, Pibarot P, et al. Discrepancies between cardiovascular magnetic resonance and Doppler echocardiography in the measurement of transvalvular gradient in aortic stenosis: the effect of flow vorticity. Journal of cardiovascular magnetic resonance. J Cardiovasc Magnetic Resonance. 2013;15(1):1.

18. John AS, Dill T, Brandt RR, Rau M, Ricken W, Bachmann G, et al. Magnetic resonance to assess the aortic valve area in aortic stenosis. JAC Elsevier Masson SAS. 2003:42(3):519-26.

19. Knobelsdorff-Brenkenhoff von $F$, Rudolph A, Wassmuth $R$, Bohl $S$, Buschmann EE, Abdel-Aty H, et al. Feasibility of cardiovascular magnetic resonance to assess the orifice area of aortic bioprostheses. Circ Cardiovasc Imaging. 2009;2(5):397-404.

20. Kupfahl C. Evaluation of aortic stenosis by cardiovascular magnetic resonance imaging: comparison with established routine clinical techniques. Heart. 2004;90(8):893-901.

21. Levy F, lacuzio L, Civaia F, Rusek S, Dommerc C, Hugues N, et al. Usefulness of 3-Tesla cardiac magnetic resonance imaging in the assessment of aortic stenosis severity in routine clinical practice. Arch Cardiovasc Dis. 2016; 109(11):618-25 Elsevier Masson SAS.

22. Malyar NM, Schlosser T, Barkhausen J, Gutersohn A, Buck T, Bartel T, et al. Assessment of aortic valve area in aortic stenosis using Cardiac magnetic resonance tomography: comparison with echocardiography. Cardiology. 2008; 109(2):126-34.

23. Maragiannis D, Jackson MS, Flores-Arredondo JH, Autry K, Schutt RC, Alvarez PA, et al. Functional Assessment of Bioprosthetic Aortic\&nbsp;Valves by CMR. J Am Coll Cardiol Img. 2016;9(7):785-93 Elsevier.

24. Mutnuru PC. Cardiac MR Imaging in the Evaluation of Rheumatic Valvular Heart Diseases. Journal of Clinical and Diagnostic Research.2016;10(3):1-4.

25. O'Brien KR, Gabriel RS, Greiser A, Cowan BR, Young AA, Kerr AJ. Aortic valve stenotic area calculation from phase contrast cardiovascular magnetic resonance: the importance of short echo time. J Cardiovasc Magn Reson. 2009;11(1):49-12..

26. Paelinck BP, Van Herck PL, Rodrigus I, Claeys MJ, Laborde JC, Parizel PM, et al. Comparison of magnetic resonance imaging of aortic valve stenosis and aortic root to multimodality imaging for selection of Transcatheter aortic valve implantation candidates. Am J Cardiol. 2011;108(1):92-8 Elsevier Inc.

27. Pouleur A-C, le Polain de Waroux J-B, Pasquet A, Vanoverschelde J-LJ, Gerber BL. Aortic valve area assessment: multidetector $C T$ compared with cine MR imaging and transthoracic and transesophageal echocardiography. Radiology. 2007:244(3):745-54.

28. Reant $\mathrm{P}$, Lederlin M, Lafitte S, Serri K, Montaudon M, Corneloup O, et al. Absolute assessment of aortic valve stenosis by planimetry using cardiovascular magnetic resonance imaging: comparison with transœsophageal echocardiography, transthoracic echocardiography, and cardiac catheterisation. Eur J Radiol. 2006;59(2):276-83.

29. Speiser U, Quick S, Haas D, Youssef A, Waessnig NK, Ibrahim K, et al. 3-T magnetic resonance for determination of aortic valve area: a comparison to echocardiography. Scand Cardiovasc J. 2014;48(3):176-83.

30. Weininger M, Sagmeister F, Herrmann S, Lange V, Schoepf UJ, Beissert M, et al. Hemodynamic assessment of severe aortic stenosis. Investig Radiol. 2011:46(1):1-10.

31. Westermann Y, Geigenmüller A, Elgeti T, Wagner M, Dushe S, Borges AC, et al. Planimetry of the aortic valve orifice area: Comparison of multislice spiral computed tomography and magnetic resonance imaging. Eur J Radiol. 2011;77(3):426-35 Elsevier Ireland Ltd.

32. Jabbour A, Ismail TF, Moat N, Gulati A, Roussin I, Alpendurada F, et al. Multimodality imaging in Transcatheter aortic valve implantation and postprocedural aortic regurgitation. JAC. 2011;58(21):2165-73 Elsevier Inc.

33. Achenbach S, Delgado V, Hausleiter J, Schoenhagen P, Min JK, Leipsic JA. SCCT expert consensus document on computed tomography imaging before transcatheter aortic valve implantation (TAVI)/transcatheter aortic valve replacement (TAVR). J Cardiovasc Computed Tomography. 2012;6(6): 366-80 Mosby, Inc.

34. Chin CWL, Khaw HJ, Luo E, Tan S, White AC, Newby DE, et al. Echocardiography underestimates stroke volume and aortic valve area: implications for patients with small-area low-gradient aortic stenosis. Can J Cardiol. 2014;30(9):1064-72 Canadian Cardiovascular Society.

35. Clavel M-A, Malouf J, Messika-Zeitoun D, Araoz PA, Michelena HI, EnriquezSarano M. Aortic valve area calculation in aortic stenosis by $C T$ and Doppler echocardiography. JACC Cardiovasc Imaging. 2015;8(3):248-57.

36. Whitener G, Sivak J, Akushevich I, Samad Z, Swaminathan M. Grading aortic stenosis with mean gradient and aortic valve Area_a comparison between preoperative transthoracic and Precardiopulmonary bypass transesophageal echocardiography. YJCAN. 2016;30(5):1254-9 Elsevier.

37. Bahlmann E, Cramariuc D, Gerdts E, Gohlke-Baerwolf C, Nienaber CA, Eriksen $E$, et al. Impact of pressure recovery on echocardiographic assessment of asymptomatic aortic stenosis: a SEAS substudy. J Am Coll Cardiol Img. 2010; 3(6):555-62.

38. Weininger M, Sagmeister F, Herrmann S, Lange V, Schoepf UJ, Beissert M, et al. Hemodynamic assessment of severe aortic stenosis: MRI evaluation of dynamic changes of vena contracta. Investig Radiol. 2011:46(1):1-10.

39. Blanken CPS, Farag ES, Boekholdt SM, Leiner T, Kluin J, Nederveen AJ, et al. Advanced cardiac MRI techniques for evaluation of left-sided valvular heart disease. J Magn Reson Imaging. 2018;48(2):318-29.

40. Sherif MA, Ince H, Maniuc O, Reiter T, Voelker W, Ertl G, et al. Twodimensional transesophageal echocardiography for aortic annular sizing in patients undergoing transcatheter aortic valve implantation. BMC Cardiovasc Disord. 2014; 14:1. 2015;15(1):181 BioMed Central.

41. Pontone G, Andreini D, Bartorelli AL, Bertella E, Mushtaq S, Gripari P, et al. Comparison of accuracy of aortic root annulus assessment with Cardiac magnetic resonance versus echocardiography and multidetector computed tomography in patients referred for Transcatheter aortic valve implantation. Am J Cardiol. 2013;112(11):1790-9 Elsevier Inc.

42. Azevedo CF, Nigri M, Higuchi ML, Pomerantzeff PM, Spina GS, Sampaio RO, et al. Prognostic significance of myocardial fibrosis quantification by histopathology and magnetic resonance imaging in patients with severe aortic valve disease. JAC. 2010;56(4):278-87 Elsevier Inc.

43. Myerson SG. Heart valve disease: investigation by cardiovascular magnetic resonance. Journal of Cardiovascular Magnetic Resonance. 2012;14(1):1-23.

44. Joziasse IC, Vink A, Cramer MJ, van Oosterhout MFM, van Herwerden LA, Heijmen R, et al. Bicuspid stenotic aortic valves: clinical characteristics and morphological assessment using MRI and echocardiography. Neth Heart J. 2011;19(3):119-25.

45. Lee SC, Ko SM, Song MG, Shin JK, Chee HK, Hwang HK. Morphological assessment of the aortic valve using coronary computed tomography 
angiography, cardiovascular magnetic resonance, and transthoracic echocardiography: comparison with intraoperative findings. Int J Cardiovasc Imaging. 2012;28(S1):33-44.

46. Figtree GA, Lønborg J, JACC SG. Cardiac magnetic resonance imaging for the interventional cardiologist. Interventionsonlinejaccorg; 2011.

47. Sherrah AG, Grieve SM, Jeremy RW, Bannon PG, Vallely MP, Puranik R. MRI in chronic aortic dissection: a systematic review and future directions. Front Cardiovasc Med Frontiers. 2015;2(2):149.

48. Sherrah AG, Callaghan FM, Puranik R, Jeremy RW, Bannon PG, Vallely MP, et al. Multi-Velocity Encoding Four-Dimensional Flow Magnetic Resonance Imaging in the Assessment of Chronic Aortic Dissection. Aorta (Stamford). 2017;5(3):80-90 Thieme Medical Publishers.

49. Kim HK, Gottliebson W, Hor K, Backeljauw P, Gutmark-Little I, Salisbury SR, et al. Cardiovascular anomalies in turner syndrome: Spectrum, prevalence, and Cardiac MRI findings in a pediatric and Young adult population. Am J Roentgenol. 2011;196(2):454-60.

50. Caruthers SD. Practical value of Cardiac magnetic resonance imaging for clinical quantification of aortic valve stenosis: comparison with echocardiography. Circulation. 2003;108(18):2236-43.

51. O'Brien KR, Cowan BR, Jain M, Stewart RAH, Kerr AJ, Young AA. MRI phase contrast velocity and flow errors in turbulent stenotic jets. J Magn Reson Imaging. 2008;28(1):210-8.

52. Falahatpisheh A, Rickers C, Gabbert D, Heng EL, Stalder A, Kramer H-H, et al. Simplified Bernoulli's method significantly underestimates pulmonary transvalvular pressure drop. J Magn Reson Imaging. 2015;43(6):1313-9

53. Mohiaddin RH, Gatehouse PD, Henien M, Firmin DN. Cine MR fourier velocimetry of blood flow through cardiac valves: comparison with Doppler echocardiography. J Magn Reson Imaging. 1997;7:657-63.

\section{Publisher's Note}

Springer Nature remains neutral with regard to jurisdictional claims in published maps and institutional affiliations.

Ready to submit your research? Choose BMC and benefit from:

- fast, convenient online submission

- thorough peer review by experienced researchers in your field

- rapid publication on acceptance

- support for research data, including large and complex data types

- gold Open Access which fosters wider collaboration and increased citations

- maximum visibility for your research: over $100 \mathrm{M}$ website views per year

At $\mathrm{BMC}$, research is always in progress.

Learn more biomedcentral.com/submissions 